Original Research Paper

\title{
Approximate Series Solution of Nonlinear, Fractional Klein- Gordon Equations Using Fractional Reduced Differential Transform Method
}

\author{
${ }^{1}$ Eman Abuteen, ${ }^{2}$ Asad Freihat, ${ }^{2}$ Mohammed Al-Smadi, ${ }^{3}$ Hammad Khalil and ${ }^{3}$ Rahmat Ali Khan \\ ${ }^{1}$ Department of Applied Science, Faculty of Engineering Technology, Al-Balqa Applied University, Amman 11942, Jordan \\ ${ }^{2}$ Department of Applied Science, Ajloun College, Al-Balqa Applied University, Ajloun 26816, Jordan \\ ${ }^{3}$ Department of Mathematics, University of Malakand, Chakadara Dir (L), Khyber Pakhtunkhwa, Pakistan
}

Article history

Received: 17-10-2015

Revised: 21-03-2016

Accepted: 24-03-2016

Corresponding Author: Mohammed Al-Smadi Department of Applied Science, Ajloun College, AlBalqa Applied University, Ajloun 26816, Jordan Email:mhm.smadi@yahoo.com

\begin{abstract}
This analysis proposes an analytical-numerical approach for providing solutions of a class of nonlinear fractional Klein-Gordon equation subjected to appropriate initial conditions in Caputo sense by using the Fractional Reduced Differential Transform Method (FRDTM). This technique provides the solutions very accurately and efficiently in convergent series formula with easily computable coefficients. The behavior of the approximate series solution for different values of fractional-order $\alpha$ is shown graphically. A comparative study is presented between the FRDTM and Implicit Runge-Kutta approach to illustrate the efficiency and reliability of the proposed technique. Our numerical investigations indicate that the FRDTM is simple, powerful mathematical tool and fully compatible with the complexity of such problems.
\end{abstract}

Keywords: Nonlinear Partial Differential Equation, Fractional Calculus, Series Solution, Fractional Reduced Differential Transform Method, Caputo Time-Fractional Derivative

\section{Introduction}

Fractional Partial Differential Equations (FPDEs) are widely used in interpretation and modeling of many of realism matters appear in applied mathematics and physics including fluid mechanics, electrical circuits, diffusion, damping laws, relaxation processes, mathematical biology (Klimek, 2005; Kilbas et al., 2010; Baleanu et al., 2009; Jumarie, 2009; Ortigueira, 2010; Mainardi, 2010). Fractional derivatives provide more accurate models of real-world problems than integer-order derivatives; they are actually found to be a powerful tool to describe certain physical problems. The topic of fractional calculus is a significantly important, useful branch of mathematics, plays a critical and serious role to describe a complex dynamical behavior in tremendous scope of application fields, helps to understand the nature of matter as well as simplified the controlling design without any loss of hereditary behaviors and explain even more complex structures.

Consider the following nonlinear Klein-Gordon equations of one-dimensional time fractional model:

$$
\begin{aligned}
& \frac{\partial^{2 \alpha} u(x, t)}{\partial t^{2 \alpha}}-\frac{\partial^{2} u(x, t)}{\partial x^{2}}+a u(x, t) \\
& +b G(u(x, t))=f(x, t), t \geq 0
\end{aligned}
$$

with initial conditions:

$$
u(x, 0)=g_{0}(x), \frac{\partial}{\partial t} u(x, 0)=g_{1}(x)
$$

where, $a$ and $b$ are real constants, $f(x, t), g_{0}(x)$ and $g_{1}(x)$ are known analytical functions, $G(u)$ is a nonlinear function, $u$ is an unknown function of $x$ and $t$ to be determined. This model is derived from well-known Klein-Gordon Equations (KGEs) by replacing the time order derivative with fractional derivative of order $\alpha$.

The KGEs are fundamental class of nonlinear evolution equations arising in classical relativistic and quantum mechanics. It got a lot of attention for studying solitons and condensed matter physics (Yusufoglu, 2008; Sweilam et al., 2012). On the other hand, analyticalnumerical studies of the solution for the FKGEs with the Caputo or Riemann-Liouville fractional derivative were 
used to handle these problems (Golmankhaneh and Baleanu, 2011; Gepreel and Mohamed, 2013; Yang et al., 2014; Marasi and Karimi, 2014; Khader et al., 2014). As we know, there is no classical method to handle the nonlinear FPDEs and provide its explicit solution due to the complexities of fractional calculus involving these equations. For this reason, we need a reliable numerical approach to find the coefficients of the fractional series solutions of such equations. During the past few decades, many numerical-analytical methods were developed for handling the FPDEs and their system. For examples of these methods, we refer to the work in (Hesameddini and Fotros, 2012; Moaddy et al., 2011; Abdulaziz et al., 2008; Hashim et al., 2009; Odibat and Momani, 2006; Khalil et al., 2015a; 2015b; 2015c; El-Ajou et al., 2015; Abu-Gdairi et al., 2015; Freihat and Al-Smadi, 2013; Momani et al., 2014; Al-Smadi et al., 2013; 2015; 2016). On the other hand, many applications for different problems by using other numerical algorithms can be found in (Abu Arqub et al., 2012; 2013; 2015; Abu Arqub and Al-Smadi, 2014; Moaddy et al., 2015; Komashynska and Al-Smadi, 2014; Komashynska et al., 2016).

In this analysis, we intend the application of FRDTM to provide numerical analytical solutions for a class of nonlinear partial differential equations included some well-known fractional Klein-Gordon equations. The FRDTM has several advantages for dealing directly with suggested equations; it needs a few iterations to get high accuracy, it is very simple for obtaining analyticalapproximate solutions in rapidly convergent formulas, it allows better significantly information in providing continuous representation of these approximations and it has the ability for solving other problems appearing in several scientific fields.

This article is organized as follows: In the next section, necessary details and preliminaries about the fractional calculus theory are briefly provided. In section 3 , the procedure of the RDTM is presented to construct and predict the series solution for fractional PDEs (1). In section 4 , numeric results for certain types of FKGEs are given to verify the validity and performance of the present method. Finally, this article ends with some concluding remarks.

\section{Mathematical Preliminaries}

The basic preliminaries, concepts and notations of fractional integrals and derivatives in Caputo definition (Caputo, 1967) are introduced as follows. Here, we adopt the Caputo fractional derivative, which is a modification of Riemann-Liouville, because the initial conditions that defined during the formulation of the system are similar to those conventional conditions of integer order.

\section{Definition 1}

A real function $u(x, t), x \in \mathbb{R}, t>0$ is said to be in the space $C_{\mu}, \mu \in \mathbb{R}$, if there exists a real number $q>\mu$ such that $u(x, t)=t^{q} u_{1}(x, t)$, where $u_{1}(x, t) \in C(\mathbb{R} \times[0, \infty))$ and it is said to be in the space $C_{\mu}^{m}$ if $\frac{\partial^{m}}{\partial t^{m}} u(x, t) \in C_{\mu}, m \in \mathbb{N}$.

\section{Definition 2}

The Riemann-Liouville integral operator of order $\alpha \geq 0$ of a function $u(x, t) \in C \mu, \mu \geq-1$ is defined as:

$$
J_{t}^{\alpha} u(x, t)= \begin{cases}\frac{1}{\Gamma(\alpha)} \int_{0}^{t}(t-\xi)^{\alpha-1} u(x, \xi) d \xi, & \alpha>0,0<\xi<t \\ u(x, t), & \alpha=0\end{cases}
$$

Consequently, the operator $J_{t}^{\alpha}$ has the following properties: For $u(x, t) \in C_{\mu}, \mu \geq-1, \alpha, \beta \geq 0, c \in \mathbb{R}$ and $\gamma>$ -1 , one can get:

- $\quad J_{t}^{\alpha} J_{t}^{\beta} u(x, t)=J_{t}^{\alpha+\beta} u(x, t)=J_{t}^{\beta} J_{t}^{\alpha} u(x, t)$

- $J_{t}^{\alpha} c=\frac{c}{\Gamma(\alpha+1)} t^{\alpha}$

- $J_{t}^{\alpha} t^{\gamma}=\frac{\Gamma(\gamma+1)}{\Gamma(\alpha+\gamma+1)} t^{\alpha+\gamma}$

Now, we introduce a modified fractional differential operator $D_{t}^{\alpha}$ proposed by Caputo as follows:

$$
D_{t}^{\alpha} f(x)=J_{t}^{m-\alpha}(x)=\frac{1}{\Gamma(m-\alpha)} \int_{t}^{x}(x-\eta)^{m-\alpha-1} f^{(m)}(\eta) d \eta, t \geq 0
$$

For $m-1<\alpha \leq m, m \in \mathbb{N}, x \geq t$ and $f x \in C_{-1}^{m}$.

\section{Definition 3}

For $m$ to be the smallest integer that exceeds $\alpha$, the Caputo time-fractional derivative operator of order $\alpha>0$ is defined as:

$$
\begin{aligned}
& D_{t}^{\alpha} u(x, t)=\frac{\partial^{\alpha} u(x, t)}{\partial t^{\alpha}} \\
& =\left\{\begin{array}{l}
J_{t}^{m-\alpha}\left(\frac{\partial^{m} u(x, t)}{\partial t^{m}}\right), 0 \leq m-1<\alpha<m \\
\frac{\partial^{m} u(x, t)}{\partial t^{m}}, \quad \alpha=m \in \mathbb{N}
\end{array}\right.
\end{aligned}
$$

\section{Theorem 1}

If $m-1<\alpha \leq m, m \in \mathbb{N}, u(x, t) \in C_{\gamma}^{m}$ and $\gamma \geq-1$, then $D_{t}^{\alpha} J_{t}^{\alpha} u(x, t)=u(x, t) \quad$ and $\quad J_{t}^{\alpha} D_{t}^{\alpha} u(x, t)=u(x, t)$ $-\sum_{k=0}^{m-1} \frac{\partial^{k} u\left(x, 0^{+}\right)}{\partial t^{k}} \frac{t^{k}}{k !}$, where $t>0$. 
For more details about FDEs, see (Millar and Ross, 1993; Podlubny, 1999; Samko et al., 1993).

\section{Description of the Method}

Let $u(x, t)$ be a function of two variables such that $u(x, t)$ $=f(x) g(t)$, then from the properties of the one-dimensional Differential Transform (DT) method, we have:

$$
u(x, t)=\sum_{i=0}^{\infty} f(i) x^{i} \sum_{j=0}^{\infty} g(j) t^{j}=\sum_{i=0}^{\infty} \sum_{j=0}^{\infty} U(i, j) x^{i} t^{j}
$$

where $U(i, j)=f(i) g(j)$ is called the spectrum of $u(x, t)$.

Next, we assume that $u(x, t)$ is continuously differentiable function with respect to space variable $x$ and time $t$.

\section{Lemma 1 (Srivastava et al., 2013)}

Let $u(x, t)$ be an analytic function, then the FRDT of $u$ is given by:

$$
U_{k}(x)=\frac{1}{\Gamma(k \alpha+1)}\left[\frac{\partial^{k \alpha} u(x, t)}{\partial t^{k \alpha}}\right] t=t_{0}
$$

where $\alpha$ is a parameter describing the order of timefractional derivative in Caputo sense.

The inverse transformed of $U_{k}$ is defined by:

$$
u(x, t)=\sum_{k=0}^{\infty} U_{k}(x)\left(t-t_{0}\right)^{k \alpha}
$$

From Equations 5 and 6, we have that:

$$
u(x, t)=\sum_{k=0}^{\infty} \frac{1}{\Gamma(k \alpha+1)}\left[\frac{\partial^{k \alpha} u(x, t)}{\partial t^{k \alpha}}\right]_{t=t_{0}}\left(t-t_{0}\right)^{k \alpha}
$$

In particular, for $t=0$, Equation 7 reduces to:

$$
u(x, t)=\sum_{k=0}^{\infty} \frac{1}{\Gamma(k \alpha+1)}\left[\frac{\partial^{k \alpha} u(x, t)}{\partial t^{k \alpha}}\right]_{t=t_{0}} t^{k \alpha}
$$

Moreover, if $\alpha=1$, then the FRDT of Equation 7 reduces to the classical RDT method.

From the above lemma, the fundamental operations of the FRDTM are given by the following theorems (Srivastava et al., 2014):

\section{Theorem 2}

Let $u(x, t), v(x, t)$ and $w(x, t)$ be any analytic functions such that $u(x, t)=R_{D}^{-1}\left[U_{k}(x)\right], v(x, t)=R_{D}^{-1}\left[V_{k}(x)\right]$ and $w(x, t)=R_{D}^{-1}\left[W_{k}(x)\right]$, then:
- $\quad$ If $u(x, t)=v(x, t) \pm w(x, t)$, then $U_{k}(x)=V_{k}(x) \pm W_{k}(x)$

- If $u(x, t)=a v(x, t)$, then $U_{k}\left(\mathrm{x}_{\mathrm{x}}\right)=a V_{k}(x), a$ is an arbitrary constant

- If $u(x, t)=x^{m} t^{n} v(x, t)$, then $U_{k}(x)=V_{k-n}(x)$

- If $u(x, t)=x^{m} t^{n}$, then $U_{k}\left({ }_{\mathrm{x}}\right)=x^{m} \delta(k-n), \delta(k)=$ $\left\{\begin{array}{l}1, k=0 \\ 0 k \neq 0\end{array}\right.$

- If $u(x, t)=v(x, t) w(x, t)$, then $U_{k}(x)=$ $\sum_{r=0}^{k} V_{r}(x) W_{k-r}(x)=\sum_{r=0}^{k} W_{r}(x) V_{k-r}(x)$

\section{Theorem 3}

Let $u(x, t)$ and $v(x, t)$ be any two analytic functions such that $u(x, t)=R_{D}^{-1}\left[U_{k}(x)\right]$ and $v(x, t)=R_{D}^{-1}\left[V_{k}(x)\right]$, then:

- If $u(x, t)=\frac{\partial^{r}}{\partial x^{r}} v(x, t)$, then $U_{k}(x)=\frac{\partial^{r}}{\partial x^{r}} V_{k}(x)$

- If $u(x, t)=\frac{\partial^{r \partial}}{\partial t^{r \partial}} v(x, t)$, then $U_{k}(x)=$ $\frac{\Gamma(\alpha k+\alpha r+1)}{\Gamma(\alpha k+1)} V_{k+r}(x)$

\section{Corollary 1}

$$
\text { If } u(x, t)=\mathrm{e}^{\lambda^{t+\mu x}}, \text { then } U_{k}(x)=\frac{\lambda^{k}}{k !} e^{\mu x} .
$$

\section{Corollary 2}

$$
\text { If } u(x, t)=\sin (\eta x+\omega t), v(x, t)=\cos (\eta x+\omega t) \text {, then } \mathrm{U}_{\mathrm{k}}(x)
$$$$
=\frac{\omega^{k}}{k !} \sin \left(\eta x+\frac{\pi k}{2}\right) \text { and } V_{k}(x)=\frac{\omega^{k}}{k !} \cos \left(\eta x+\frac{\pi k}{2}\right) \text {. }
$$

The reader is referred to (Keskin and Oturanc, 2009; Abazari and Abazari, 2012; Secer, 2012; Sohail and Mohyud-Din, 2012; Al-Amr, 2014) and the references therein to know more details about the reduced differential transform technique, including their applications in various kinds of differential equations.

Now, by applying the FRDTM to Equation 1, we obtain the following recurrence relation formula:

$$
\begin{aligned}
& \frac{\Gamma(\alpha k+2 \alpha+1)}{\Gamma(\alpha k+1)} U_{k+2}(x)=\frac{\partial^{2}}{\partial x^{2}} U_{k}(x) \\
& -a U_{k}(x)-b G\left[U_{k}(x)\right]+F_{k}(x)
\end{aligned}
$$

where $F_{k}(x)$ and $G\left[U_{k}(x)\right]$ are the reduced transformation of the functions $f(x, t)$ and $G(u(x, t))$, respectively.

Using the initial conditions, we have:

$$
U_{0}(x)=g_{0}(x), U_{1}(x)=g_{1}(x)
$$

Substituting Equation 10 into Equation 9 and by straightforward iterative calculation, we obtain the 
values $U_{k}(x)$, for $k=1,2,3, \ldots$. Thus, inverse RDT of $\left\{U_{k}(x)\right\}_{k=1}^{n}$ yields that:

$$
\breve{u}_{n}(x, t)=\sum_{k=0}^{n} U_{k}(x)\left(t-t_{0}\right)^{k \alpha}
$$

Therefore, the closed form solution given by:

$$
u(x, t)=\lim _{x \rightarrow \infty} \breve{u}_{n}(x, t)
$$

\section{Numerical Examples}

In this section, some numerical examples are given to verify the simplicity and applicability of the present technique in finding approximate series solution for fractional KGEs. The simulation results indicate that the FRDT method is highly accurate and fully compatible with the complexity of the PDEs of fractional-order.

\section{Example 4.1}

We consider the following one-dimensional linear fractional Klein-Gordon equation:

$$
\frac{\partial^{\alpha} u(x, t)}{\partial t^{\alpha}}-\frac{\partial^{2} u(x, t)}{\partial x^{2}}-u(x, t)=0,0<\alpha \leq 1
$$

with initial condition:

$$
u(x, 0)=1+\sin (x)
$$

where $x, t \geq 0$.

Applying the transformation ( $\mathrm{Li}$ and $\mathrm{He}, 2010$ ), we have:

$$
\frac{\partial u(x, t)}{\partial T}-\frac{\partial^{2} u(x, t)}{\partial x^{2}}=u(x, t)
$$

By using the FRDTM of Equation 13, we have the recurrence relation formula:

$$
U_{k+1}(x)=\frac{\Gamma(\alpha k+1)}{\Gamma(\alpha(k+1)+1)}\left[\frac{\partial^{2}}{\partial x^{2}} U_{k}(x)+U_{k}(x)\right]
$$

with transformed initial data:

$$
U_{0}(x)=1+\sin (x)
$$

Substituting the condition (15) into Equation 14, we get the values successively $U_{k}(x), k=1,2,3, \ldots$, as follows:

$$
\begin{aligned}
& U_{1}(x)=\frac{1}{\Gamma(\alpha+1)}, U_{2}(x)=\frac{1}{\Gamma(2 \alpha+1)}, \\
& U_{3}(x)=\frac{1}{\Gamma(3 \alpha+1)}, \ldots, U_{k}(x)=\frac{1}{\Gamma(k \alpha+1)}
\end{aligned}
$$

Thus, the approximate solution can be obtained by:

$$
\begin{aligned}
& u(x, t)=1+\sin (x)+\frac{1}{\Gamma(\alpha+1)} T \\
& +\frac{1}{\Gamma(2 \alpha+1)} T^{2}+\frac{1}{\Gamma(3 \alpha+1)} T^{3}+\ldots
\end{aligned}
$$

The inverse RDTM is given by:

$$
\begin{aligned}
& u(x, t)=1+\sin (x)+\frac{1}{\Gamma(\alpha+1)} t^{\alpha} \\
& +\frac{1}{\Gamma(2 \alpha+1)} t^{2 \alpha}+\frac{1}{\Gamma(3 \alpha+1)} t^{3 \alpha}+\ldots \\
& =\sin (x)+\sum_{k=0}^{\infty} \frac{1}{\Gamma(2 \alpha+1)} t^{k \alpha}
\end{aligned}
$$

Consequently, the reduced inverse transformed of $U_{k}(x)$ follows the closed form solution. Setting $\alpha=1$, the exact solution is:

$$
u(x, t)=\sin (x)+e^{t}
$$

To demonstrate the efficiency of the present method, we compare the FRDT approximation with the Implicit Runge-Kutta (IRK) method for $\alpha=1$. Figure 1 shows the phase portrait of solutions for Example 4.1 using the FRDTM and IRKM for $t \in[0,0.8]$ and $x \in[0,4]$. The numerical results for different time levels of $\alpha$ are presented in Fig. 2. Here, we note that the approximate FRDT are efficiency for time-fractional KGE and very closed to the IRK solutions.

\section{Example 4.2}

We consider the nonlinear Klein-Gordon fractional model in the form:

$\frac{\partial^{\alpha} u(x, t)}{\partial t^{\alpha}}-\frac{\partial^{2} u(x, t)}{\partial x^{2}}+u^{2}(x, t)=0,0<\alpha \leq 1$

with initial condition:

$$
u(x, 0)=1+\sin (x)
$$
have:

Applying the transformation ( $\mathrm{Li}$ and $\mathrm{He}, 2010$ ), we 


$$
\frac{\partial u(x, t)}{\partial T}-\frac{\partial^{2} u(x, t)}{\partial x^{2}}=u^{2}(x, t)
$$

By using the FRDTM, we have the recurrence relation formula:

$$
U_{k+1}(x)=\frac{\Gamma(\alpha k+1)}{\Gamma(\alpha(k+1)+1)}\left[\begin{array}{l}
\frac{\partial^{2}}{\partial x^{2}} U_{k}(x) \\
+\sum_{r=0}^{k} U_{r}(x) U_{k-r}(x)
\end{array}\right]
$$

With RDTM of initial condition:

$$
U_{0}(x)=1+\sin (x)
$$

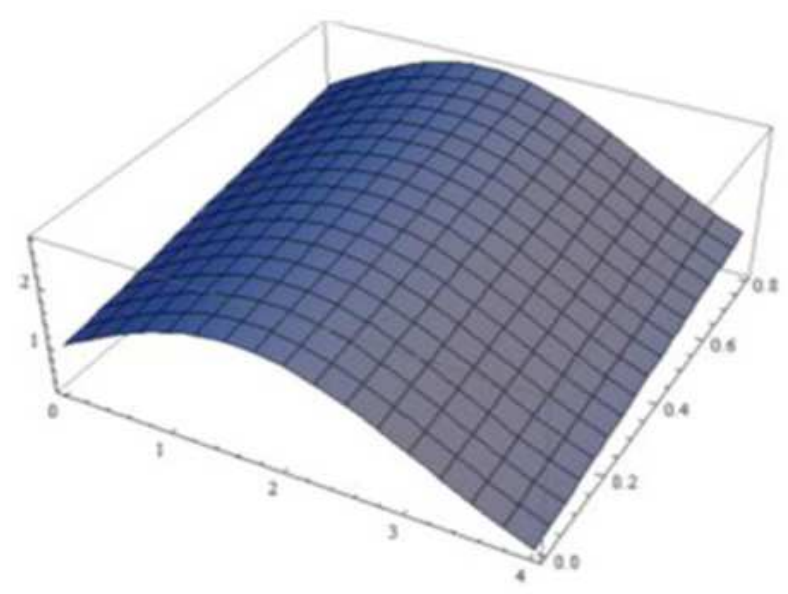

(a)
Following recurrence relation (17), the sequences components $U_{k}(x), k=1,2,3, \ldots$, were computed using the Mathematica package, can be successively given by:

$$
\begin{aligned}
& U_{1}(x)=\frac{-\Gamma(\alpha)}{\Gamma(2 \alpha)}\left[1+3 \sin (x)+\sin ^{2}(x)\right], \\
& U_{2}(x)-\frac{-\Gamma(\alpha)}{2 \Gamma(3 \alpha)}\left[\begin{array}{l}
12 \cos (2 x)-25 \sin (x) \\
+\sin (3 x)-12
\end{array}\right], \\
& U_{3}(x)=\frac{-\Gamma(\alpha)}{8(\Gamma(2 \alpha))^{2} \Gamma(4 \alpha)}\left[\begin{array}{l}
2 \Gamma(\alpha) \Gamma(3 \alpha) \\
\left(\begin{array}{l}
\left.-3+\cos (2 x)-6 \sin ^{2}(x)\right)+4(\Gamma(2 \alpha))^{2} \\
49-98 \cos (2 x) \\
+\cos (4 x)+111 \sin (x)-23 \sin (3 x)
\end{array}\right)
\end{array}\right], \\
& \vdots \\
& \text { and soon }
\end{aligned}
$$

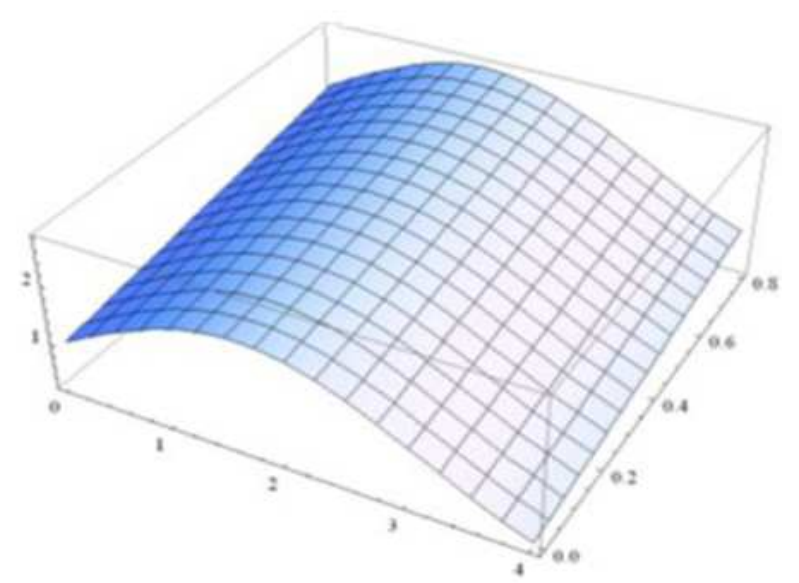

(b)

Fig. 1. Comparison of phase plot for $u(x, t)$ of Example 4.1 at $\alpha=1, x \in[0,4]$ and $t \in 0,0.8$ : (a) the FRDTM; (b) implicit Runge-Kutta method

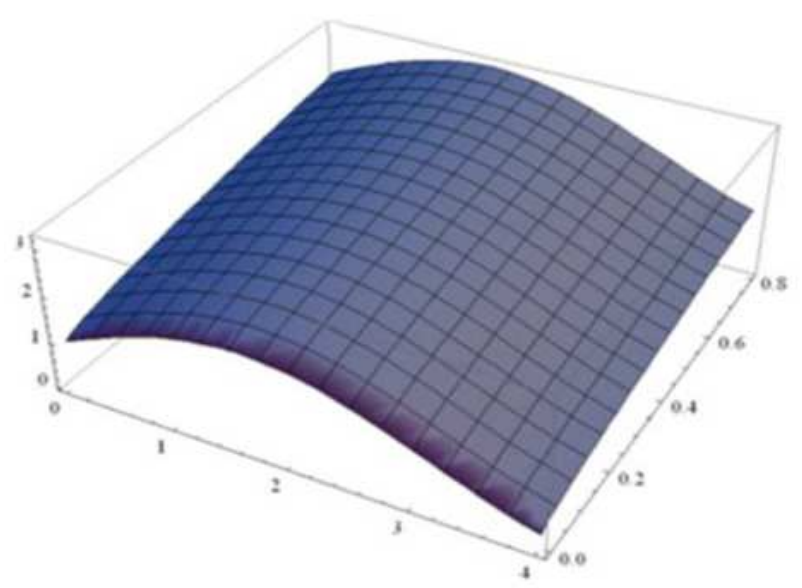

(a)

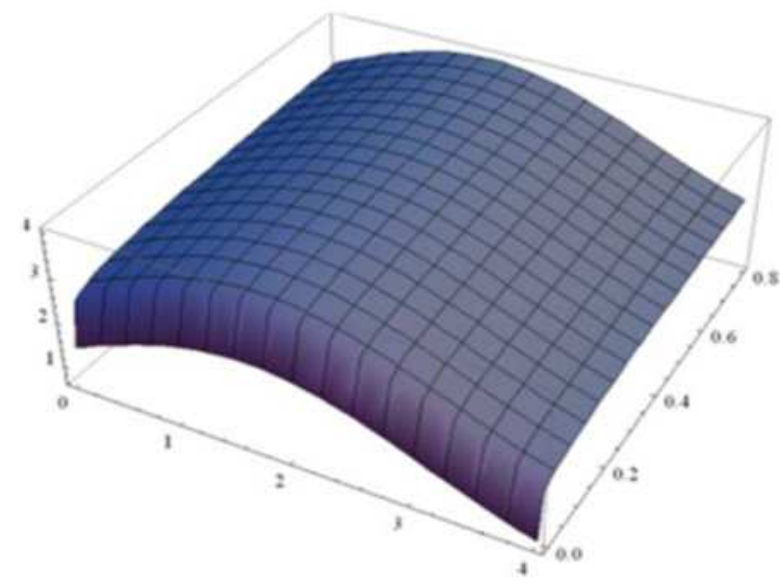

(b)

Fig. 2. Phase plot of the solution $u(x, t)$ of Example 4.1 using the FRDTM for $x \in[0,4]$ and $t \in[0,0.8]:$ (a) at $\alpha=0.7$; (b) at $\alpha=0.1$ 
The approximate form solution is given by:

$u(x, t)=1+\sin (x)+U_{1}(x) T+U_{2}(x) T^{2}+U_{3}(x) T^{3}+\ldots$

Accordingly, the inverse RDT is given by:

$$
\begin{aligned}
& u(x, t)=\sum_{k=0}^{\infty} U_{k}(x) t^{k \alpha} \\
& =1+\sin (x)-\frac{\Gamma(\alpha)}{\Gamma(2 \alpha)}\left[1+3 \sin (x)+\sin ^{2}(x)\right] t^{\alpha} \\
& -\frac{\Gamma(\alpha)}{2 \Gamma(3 \alpha)}[12 \cos (2 x)-25 \sin (x)+\sin (3 x)-12] t^{2 \alpha} \\
& -\frac{\Gamma(\alpha)}{8(\Gamma(2 \alpha))^{2} \Gamma(4 \alpha)}\left[\begin{array}{l}
2 \Gamma(\alpha) \Gamma(3 \alpha)\left(\begin{array}{l}
-3+\cos (2 x) \\
-6 \sin ^{2}(x)
\end{array}\right) \\
\left.+4(\Gamma(2 \alpha))^{2}\left(\begin{array}{l}
49-98 \cos (2 x)+\cos (4 x) \\
+111 \sin (x)-23 \sin (3 x)
\end{array}\right)\right] t^{3 \alpha} \\
+\cdots .
\end{array}\right.
\end{aligned}
$$

The series solution of $u$ follows closed form solution.

The geometric behaviors of the solution for Example 4.2 are studied by drawing the 3-dimensional space

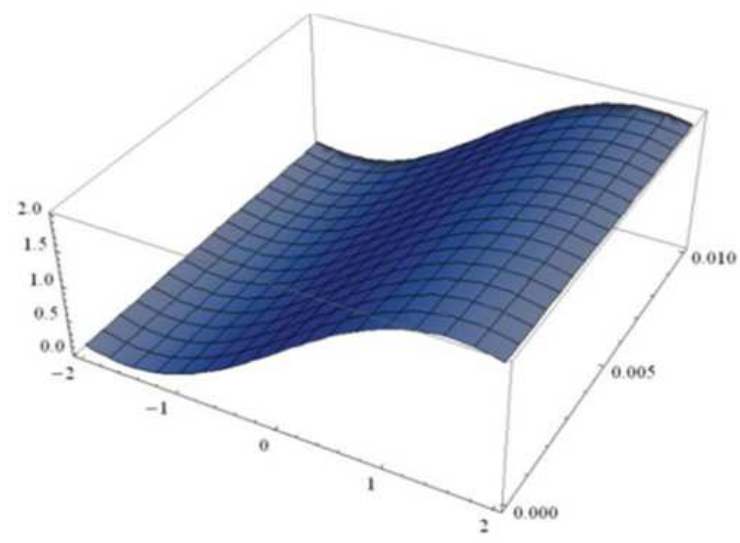

(a) figures of the FRDT approximate solution together with its corresponding IRK solution. Figure 3 shows the comparison between the FRDT approximate solution and IRK solution at $\alpha=1$ for $x \in[-2,2]$ and $t \in[0,0.01]$. Whileas, Fig. 4 shows the solution behavior of the nonlinear FKGE for different specific cases of $\alpha$ in the domain $x \in[-2,2]$ and $t \in[0,0.01]$. The performance errors analysis are obtained by the FRDTM at $x=2$ and summarized in Table 1. Numerically, it is showed that the RDT method is effective and accurate.

\section{Example 4.3}

We consider the nonlinear Klein-Gordon fractional model in the form:

$$
\frac{\partial^{\alpha} u(x, t)}{\partial t^{\alpha}}-\frac{\partial^{2} u(x, t)}{\partial x^{2}}+u(x, t)-u^{3}(x, t)=0,0<\alpha \leq 1
$$

with initial condition:

$$
u(x, 0)=-\sec h(x)
$$

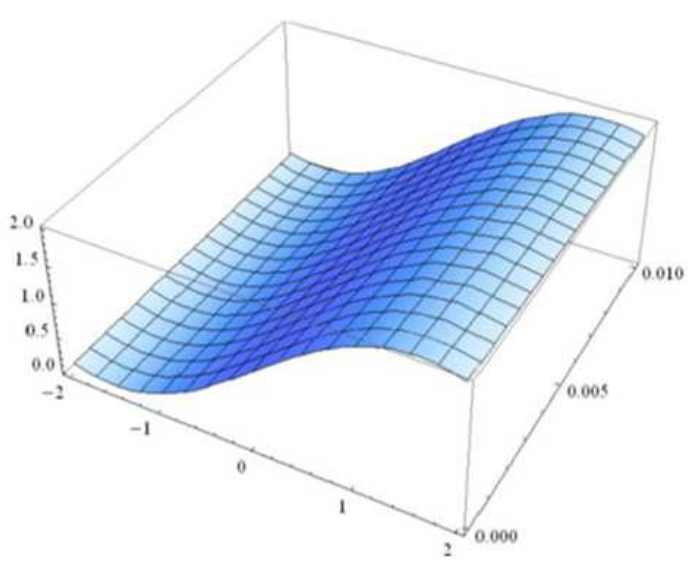

(b)

Fig. 3. Comparison of phase plot for $u(x, t)$ of Example 4.2 at $\alpha=1, x \in[-2,2]$ and $t \in 0,0.01$ : (a) the FRDTM; (b) implicit RKM

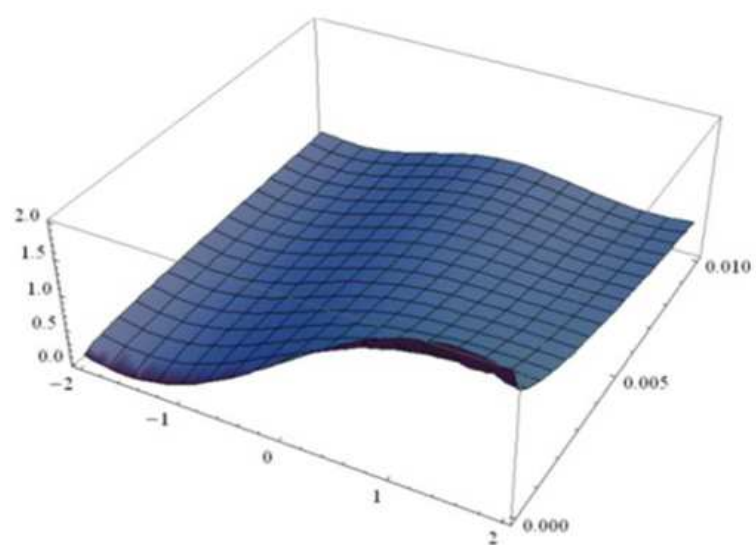

(a)

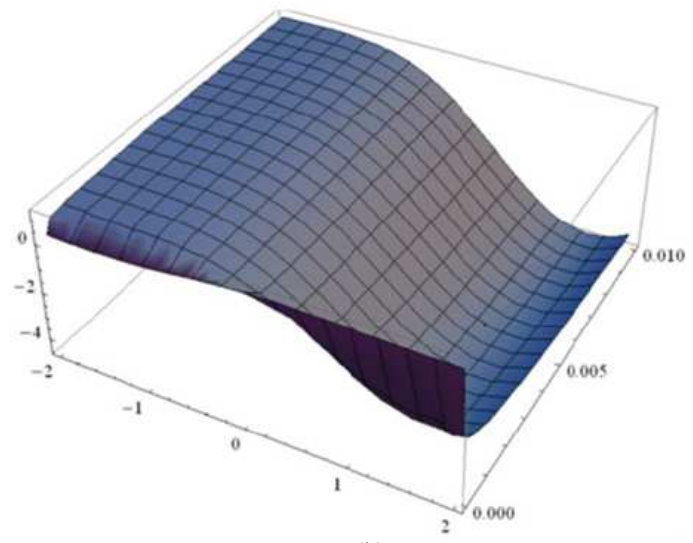

(b)

Fig. 4. Phase plot of the solution $u(x, t)$ of Example 4.2 using the FRDTM for $x \in[-2,2]$ and $t \in[0,0.01]$ : (a) at $\alpha=0.4$; (b) at $\alpha=0.1$ 
Table 1. The error analysis for Example 4.2 when $\alpha=1$ with $x=2$

\begin{tabular}{lllll}
\hline$t$ & RDTM & IRKM & Absolute error & Relative error \\
\hline 0.000 & 1.909297426825682 & 1.909297426825682 & 0.00000 & 0.00000 \\
0.001 & 1.904742712734773 & 1.904743294464910 & $5.81730 \times 10^{-7}$ & $3.05411 \times 10^{-7}$ \\
0.002 & 1.900187998643864 & 1.900189162104138 & $1.16346 \times 10^{-6}$ & $6.12287 \times 10^{-7}$ \\
0.003 & 1.895633284552955 & 1.895635029743366 & $1.74519 \times 10^{-6}$ & $9.20636 \times 10^{-7}$ \\
0.004 & 1.891078570462046 & 1.891080897382594 & $2.32692 \times 10^{-6}$ & $1.23047 \times 10^{-6}$ \\
0.005 & 1.886523856371138 & 1.886526765021823 & $2.90865 \times 10^{-6}$ & $1.54180 \times 10^{-6}$ \\
0.006 & 1.881969142280229 & 1.881972632661051 & $3.49038 \times 10^{-6}$ & $1.85464 \times 10^{-6}$ \\
0.007 & 1.877414428189319 & 1.877418500300279 & $4.07211 \times 10^{-6}$ & $2.16899 \times 10^{-6}$ \\
0.008 & 1.872859714098411 & 1.872864367939507 & $4.65384 \times 10^{-6}$ & $2.48488 \times 10^{-6}$ \\
0.009 & 1.868305000007502 & 1.868310235578735 & $5.23557 \times 10^{-6}$ & $2.80230 \times 10^{-6}$ \\
0.010 & 1.863750285916593 & 1.863756103217964 & $5.81730 \times 10^{-6}$ & $3.12128 \times 10^{-6}$ \\
\hline
\end{tabular}
have:

Applying the transformation ( $\mathrm{Li}$ and $\mathrm{He}, 2010$ ), we

$$
\frac{\partial u(x, t)}{\partial T}=\frac{\partial^{2} u(x, t)}{\partial x^{2}}-u(x, t)+u^{3}(x, t)
$$

By using the FRDTM, we have the recurrence relation formula:

$$
U_{k+1}(x)=\frac{\Gamma(\alpha k+1)}{\Gamma(\alpha(k+1)+1)}\left[\begin{array}{l}
\frac{\partial^{2}}{\partial x^{2}} U_{k}(x)-U_{k}(x) \\
+\sum_{r=0}^{k} \sum_{i=0}^{r} U_{i}(x) U_{k-r}(x)
\end{array}\right]
$$

with RDT of initial condition:

$$
U_{0}(x)=-\sec h(x)
$$

Following recurrence relation (19), the sequences components $U_{k}(x), k=1,2,3, \ldots$, were computed using the Mathematica 9.0 package, can be successively given by:

$$
\begin{aligned}
& U_{1}(x)=\frac{\Gamma(\alpha)}{\Gamma(2 \alpha)}\left[(\operatorname{sech}(x))^{3}\right], \\
& U_{2}(x)=\frac{\Gamma(\alpha)}{\Gamma(3 \alpha)}\left[(\operatorname{sech}(x))^{5}(4 \cosh (2 x)-5],\right. \\
& U_{3}(x)=\frac{\Gamma(\alpha)}{(\Gamma(2 \alpha))^{2} \Gamma(4 \alpha)}\left[(\operatorname{sech}(x))^{7}\left(\begin{array}{l}
\left(\begin{array}{l}
123-112 \cosh (2 x) \\
+8 \cosh (4 x) \\
(\Gamma(2 \alpha))^{2} 3 \Gamma(\alpha) \Gamma(3 \alpha)
\end{array}\right) \\
\vdots
\end{array}\right),\right. \\
& \text { and soon }
\end{aligned}
$$

By taking the inverse RDT of $\left\{U_{k}(x)\right\}_{k=0}^{n}$, we have:

$$
\begin{aligned}
& u(x, t)=\sum_{k=0}^{\infty} U_{k}(x) t^{k \alpha} \\
& =-\operatorname{sech}(x)+\frac{\Gamma(\alpha)}{\Gamma(2 \alpha)}(\operatorname{sech}(x))^{3} t^{\alpha} \\
& +\frac{\Gamma(\alpha)}{\Gamma(3 \alpha)}\left[(\operatorname{sech}(x))^{5}(4 \cosh (2 x)-5] t^{2 \alpha}\right. \\
& +\frac{\Gamma(\alpha)}{(\Gamma(2 \alpha))^{2} \Gamma(4 \alpha)}\left[(\operatorname{sech}(x))^{7}\left(\begin{array}{l}
123-112 \cosh (2 x) \\
+8 \cosh (4 x) \\
(\Gamma(2 \alpha))^{2}-3(\alpha) \Gamma(3 \alpha)
\end{array}\right)\right] t^{3 \alpha}+\cdots .
\end{aligned}
$$

Figure 5 and 6 show the solution behavior for the nonlinear time-fractional KGE in Example 4.3 by using the RDTM and IRKM at $x \in[-2,2]$ and $t \in 0,0.01$ for different particular values of $\alpha$.

\section{Example 4.4}

We consider the nonlinear Klein-Gordon fractional model in the from:

$$
\frac{\partial^{2 \alpha} u(x, t)}{\partial t^{2 \alpha}}-\frac{\partial^{2} u(x, t)}{\partial x^{2}}+u^{2}(x, t)=0,1<\alpha \leq 2
$$

with initial conditions:

$$
u(x, 0)=1+\sin (x), \frac{\partial}{\partial t} u(x, 0)=0
$$

Using the transformation ( $\mathrm{Li}$ and $\mathrm{He}, 2010$ ), we have:

$$
\frac{\partial u(x, t)}{\partial T}=\frac{\partial^{2} u(x, t)}{\partial x^{2}}-u^{2}(x, t)
$$

By using the FRDTM, we obtain the recurrence relations formula as: 


$$
U_{k+2}(x)=\frac{\Gamma(\alpha k+1)}{\Gamma(\alpha(k+2)+1)}\left[\begin{array}{l}
\frac{\partial^{2}}{\partial x^{2}} U_{k}(x) \\
-\sum_{r=0}^{k} U_{r}(x) U_{k-r}(x)
\end{array}\right]
$$

with RDT of initial condition:

$$
U_{0}(x)=1+\sin (x), U_{1}(x)=0
$$

Following recurrence relation straightforward iterative steps, yields:

(22) and by

$$
\begin{aligned}
& U_{2}(x)=-\frac{\Gamma(\alpha)}{\Gamma(3 \alpha)}\left[1+3 \sin (x)+\sin ^{2}(x)\right], U_{3}(x)=0, \\
& U_{4}(x)=-\frac{\Gamma(\alpha)}{2 \Gamma(4 \alpha)}[12 \cos (2 x)-25 \sin (x)+\sin (3 x)-12], U_{5}(x)=0, \\
& U_{6}(x)=-\frac{\Gamma(\alpha)}{8(\Gamma(2 \alpha))^{2} \Gamma(5 \alpha)}\left[\begin{array}{l}
2 \Gamma(\alpha) \Gamma(3 \alpha)\left(\begin{array}{l}
-3+\cos 2 x \\
-6 \sin ^{2} x
\end{array}\right) \\
+4(\Gamma(2 \alpha))^{2}\left(\begin{array}{l}
-12+12 \cos 2 x \\
-25 \sin x+\sin 3 x
\end{array}\right)
\end{array}\right], \\
& U_{7}(x)=0
\end{aligned}
$$

Table 2. Numerical results of $u$ when $x=2$ for Example 4.4

\begin{tabular}{lcccc}
\hline$t$ & $\alpha=1.25$ & $\alpha=1.5$ & $\alpha=1.75$ & $\alpha=2$ \\
\hline 0.00 & 1.9092974268257 & 1.9092974268257 & 1.9092974268257 & 1.9092974268257 \\
0.02 & 1.8577557126584 & 1.8953639720340 & 1.9056740630824 & 1.9083864840075 \\
0.04 & 1.7867098804114 & 1.8698876653513 & 1.8971099324942 & 1.9056536555530 \\
0.06 & 1.7057992950300 & 1.8368970719530 & 1.8845189771575 & 1.9010989414620 \\
0.08 & 1.6177334620125 & 1.7978297884919 & 1.8683037456485 & 1.8947223417348 \\
0.10 & 1.5239332295153 & 1.7535166664471 & 1.8487201924577 & 1.8865238563711 \\
0.12 & 1.4252945743833 & 1.7045182992625 & 1.8259529888198 & 1.8765034853711 \\
0.14 & 1.3224416173263 & 1.6512462328457 & 1.8001449727689 & 1.8646612287348 \\
0.16 & 1.2158375439572 & 1.5940193350310 & 1.7714116686258 & 1.8509970864620 \\
0.18 & 1.1058416180037 & 1.5330941474492 & 1.7398494741889 & 1.8355110585530 \\
0.20 & 0.9927417362082 & 1.4686828986574 & 1.7055407099216 & 1.8182031450075 \\
\hline
\end{tabular}

Table 3. The numerical results for Example 4.4 when $\alpha=2$ with $x=2$.

\begin{tabular}{lccll}
\hline$t$ & FRDTM & IRKM & Absolute error & Relative error \\
\hline 0.00 & 1.90929742682568 & 1.90929742682568 & 0.00000 & 0.00000 \\
0.02 & 1.90838648400750 & 1.90838660035353 & $1.16346 \times 10^{-7}$ & $6.09656 \times 10^{-8}$ \\
0.04 & 1.90565365555296 & 1.90565412093706 & $4.65384 \times 10^{-6}$ & $2.44212 \times 10^{-7}$ \\
0.06 & 1.90109894146205 & 1.90109998857629 & $1.04711 \times 10^{-6}$ & $5.50794 \times 10^{-7}$ \\
0.08 & 1.89472234173477 & 1.89472420327121 & $1.86154 \times 10^{-6}$ & $9.82484 \times 10^{-7}$ \\
0.10 & 1.88652385637114 & 1.88652676502182 & $2.90865 \times 10^{-6}$ & $1.54180 \times 10^{-6}$ \\
0.12 & 1.87650348537114 & 1.87650767382812 & $4.18846 \times 10^{-6}$ & $2.23205 \times 10^{-6}$ \\
0.14 & 1.86466122873478 & 1.86466692969012 & $5.70096 \times 10^{-6}$ & $3.05736 \times 10^{-6}$ \\
0.16 & 1.85099708646205 & 1.85100453260780 & $7.44615 \times 10^{-6}$ & $4.02276 \times 10^{-6}$ \\
0.18 & 1.83551105855296 & 1.83552048258118 & $9.42403 \times 10^{-6}$ & $5.13425 \times 10^{-6}$ \\
0.20 & 1.81820314500750 & 1.81821477961024 & $1.16346 \times 10^{-5}$ & $6.39892 \times 10^{-6}$ \\
\hline
\end{tabular}

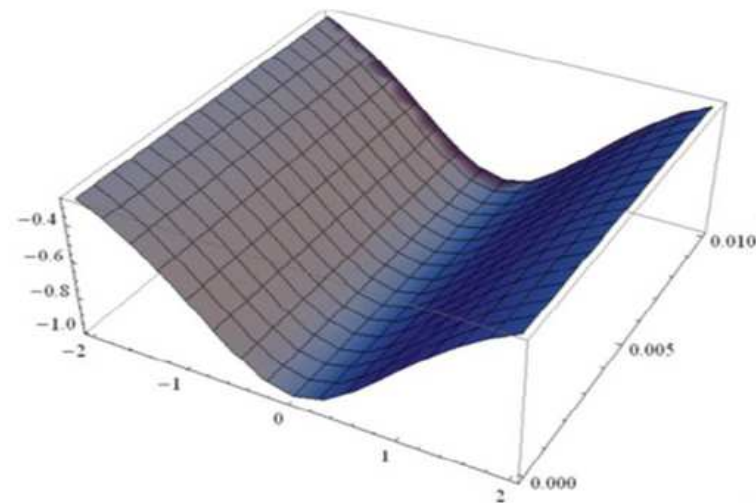

(a)

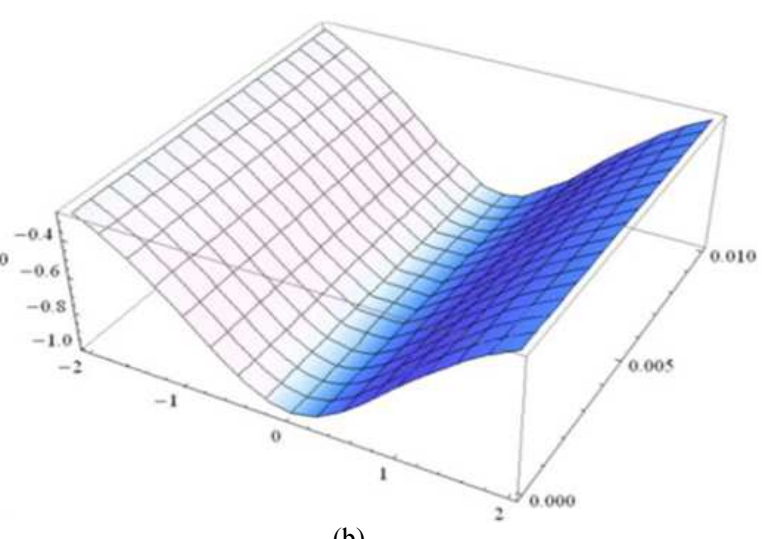

(b)

Fig. 5.Comparison of phase plot for $u(x, t)$ of Example 4.3 at $\alpha=1, x \in[-2,2]$ and $t \in[0,0.01]$ : (a) the FRDTM; (b) implicit Runge-Kutta method 


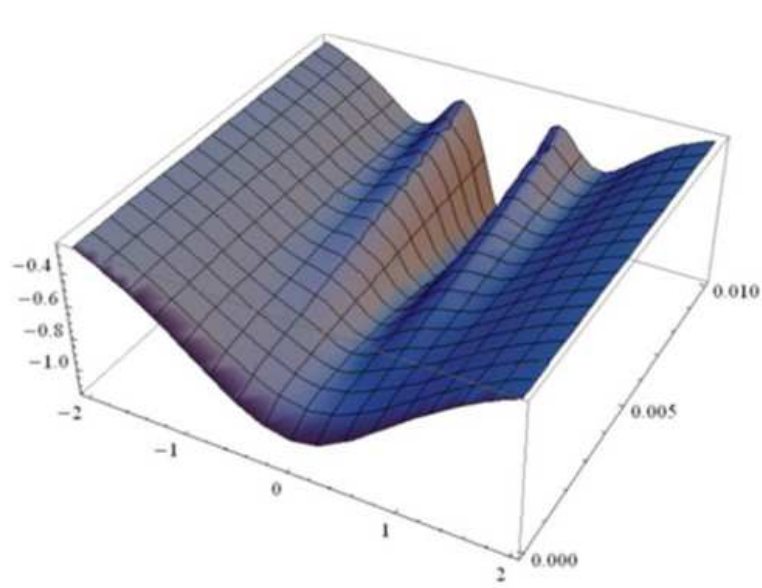

(a)

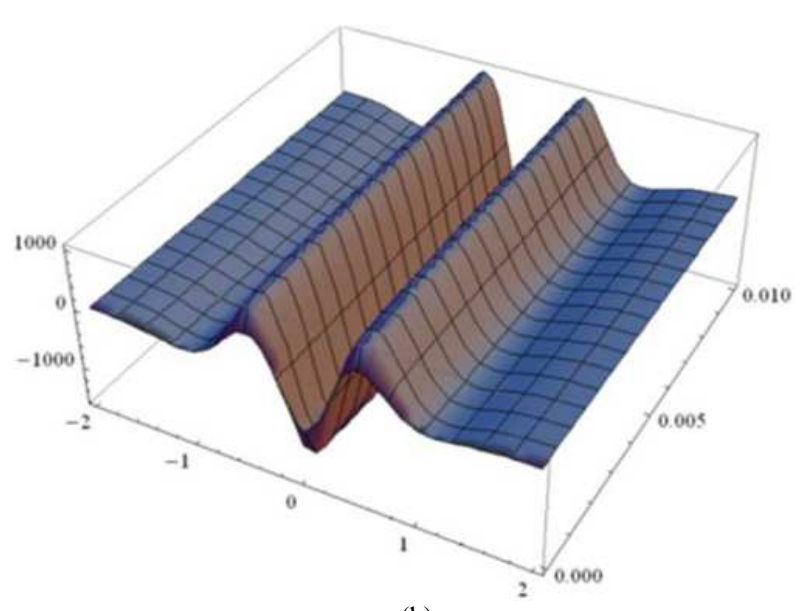

(b)

Fig. 6. Phase plot of the solution $u(x, t)$ of Example 4.2 using the FRDTM for $x \in[-2,2]$ and $t \in[0,0.01]$ : (a) at $\alpha=0.4$; (b) at $\alpha$ $=0.1$

The approximate form solution is obtained by:

$$
\begin{aligned}
& u(x, t)=1+\sin (x)+U_{1}(x) T \\
& +U_{2}(x) T^{2}+U_{3}(x) T^{3}+U_{4}(x) T^{4}+\cdots
\end{aligned}
$$

Consequently, the reduced inverse DT of $U_{k}(x)$ will be given by:

$$
\begin{aligned}
& u(x, t)=\sum_{k=0}^{\infty} U_{k}(x) t^{k \alpha} \\
& =1+\sin (x)-\frac{\Gamma(\alpha)}{\Gamma(2 \alpha)}\left[1+3 \sin (x)+\sin ^{2}(x)\right] t^{\alpha} \\
& -\frac{\Gamma(\alpha)}{\Gamma(3 \alpha)}\left[1+3 \sin (x)+\sin ^{2}(x)\right] t^{2 \alpha} \\
& -\frac{\Gamma(\alpha)}{2 \Gamma(4 \alpha)}[12 \cos (2 x)-25 \sin (x)+\sin (3 x)-12] t^{3 \alpha} \\
& -\frac{\Gamma(\alpha)}{8(\Gamma(2 \alpha))^{2} \Gamma(5 \alpha)}\left[\begin{array}{l}
2 \Gamma(\alpha) \Gamma(3 \alpha)\left(\begin{array}{l}
-3+\cos 2 x \\
-6 \sin ^{2} x
\end{array}\right) \\
+4(\Gamma(2 \alpha))^{2}\left(\begin{array}{l}
-12+12 \cos 2 x \\
-25 \sin x+\sin 3 x
\end{array}\right)
\end{array}\right] t^{4 \alpha}+\cdots
\end{aligned}
$$

In Table 2, we summarized numerical values for the solution of Equation 20 when $\alpha=1.25,1.5,1.75$ and $\alpha=2$ with $x=2$. Also numerical results using our approximation and the implicit Runge-Kutta method for $\alpha=2$ are summarized in Table 3. From these tables, it is easier to observe that the numerical approximations are in agreement with each other's and with the IRK method. Also, they have same behavior as those obtained using the implicit RungeKutta method.

\section{Concluding Remarks}

In this study, we present numerical algorithm for finding approximate form solutions of a class of KleinGordon fractional model based upon FRDTM. This method was used directly without employing linearization and perturbation. The efficiency and capability of the present algorithm have been checked via several illustrated examples. The results reveal the complete reliability of this method with a great potential in scientific applications. Finally, we conclude that the FRDTM is very powerful, straightforward and effective to obtain analytical numerical solutions of a wide variety problems related to fractional PDEs applied in mathematics, physics and engineering. Computations of this paper have been carried out by using the computer package of Mathematica 9.

\section{Acknowledgment}

The authors express their thanks to unknown referees for the careful reading and helpful comments.

\section{Author's Contributions}

All authors have contributed equally.

\section{Conflicts of Interest}

The authors declare that there is no conflict of interests regarding the publication of this paper.

\section{References}

Al-Amr, M.O., 2014. New applications of reduced differential transform method. Alex. Eng. J., 53: 243-247. DOI: 10.1016/j.aej.2014.01.003 
Abazari, R. and M. Abazari, 2012. Numerical simulation of generalized Hirota-Satsuma coupled $\mathrm{KdV}$ equation by RDTM and comparison with DTM. Comm. Nonlin. Sci. Num. Simul., 17: 619-629. DOI: $10.1016 /$ j.cnsns.2011.05.022

Abdulaziz, O., I. Hashim and A. Saif, 2008. Series solutions of time-fractional PDEs by homotopy analysis method. Differential Equat. Nonlinear Mechan., 2008: 686512-686527. DOI: $10.1155 / 2008 / 686512$

Abu Arqub, O., M. Al-Smadi and S. Momani, 2012. Application of reproducing kernel method for solving nonlinear Fredholm-Volterra integrodifferential equations. Abstract Applied Anal., 2012: 839836-839851. DOI: 10.1155/2012/839836

Abu Arqub, O., M. Al-Smadi and N. Shawagfeh, 2013. Solving Fredholm integro-differential equations using reproducing kernel Hilbert space method. Applied Math. and Comput., 219: 8938-8948. DOI: 10.1016/j.amc.2013.03.006

Abu Arqub, O. and M. Al-Smadi, 2014. Numerical algorithm for solving two-point, second order periodic boundary value problems for mixed integro-differential equations. Applied Math. Comput., 243: 911-922.

DOI: $10.1016 /$ j.amc.2014.06.063

Abu Arqub, O., M. Al-Smadi, S. Momani and T. Hayat, 2015. Numerical solutions of fuzzy differential equations using reproducing kernel hilbert space method. Soft Comput.

DOI: $10.1007 / \mathrm{s} 00500-015-1707-4$

Abu-Gdairi, R., M. Al-Smadi and G. Gumah, 2015. An expansion iterative technique for handling fractional differential equations using fractional power series scheme. J. Math. Stat., 11: 29-38.

DOI: 10.3844 /jmssp.2015.29.38

Al-Smadi, M., A. Freihat, M. Abu Hammad, S. Momani and O. Abu Arqub, 2016. Analytical approximations of partial differential equations of fractional order with multistep approach. J. Comput. Theoretical Nanosci.

Al-Smadi, M., A. Freihat, O. Abu Arqub and N. Shawagfeh, 2015. A novel multistep generalized differential transform method for solving fractionalorder Lu chaotic and hyperchaotic systems. J. Comput. Anal. Applic., 19: 713-724.

Al-Smadi, M., O. Abu Arqub and S. Momani, 2013. A computational method for two-point boundary value problems of fourth-order mixed integrodifferential equations. Math. Problems Eng., 2013: 832074-832083. DOI: 10.1155/2013/832074

Baleanu, D., A.K. Golmankhaneh and M.C. Baleanu, 2009. Fractional electromagnetic equations using fractional forms. Int. J. Theor. Phy., 48: 3114-3123. DOI: $10.1007 / \mathrm{s} 10773-009-0109-8$
Caputo, M., 1967. Linear models of dissipation whose Q is almost frequency independent-II. Geophys. J. Int., 13: 529-539. DOI: 10.1111/j.1365-246X.1967.tb02303.x

El-Ajou, A., O. Abu Arqub and M. Al-Smadi, 2015. A general form of the generalized Taylor's formula with some applications. Applied Math. Comp., 256: 851-859. DOI: 10.1016/j.amc.2015.01.034

Freihat, A. and M. Al-Smadi, 2013. A new reliable algorithm using the generalized differential transform method for the numeric-analytic solution of fractional-order Liu chaotic and hyperchaotic systems. Pensee J., 75: 263-276.

Gepreel, K.A. and M.S. Mohamed, 2013. Analytical approximate solution for nonlinear space-time fractional Klein-Gordon equation. Chin. Phys. B. DOI: 10.1088/1674-1056/22/1/010201

Golmankhaneh, A.K. and D. Baleanu, 2011. On nonlinear fractional Klein-Gordon equation. Sign. Proces., 91: 446-451. DOI: $10.1016 /$ j.sigpro.2010.04.016

Hashim, I., O. Abdulaziz and S. Momani, 2009. Homotopy analysis method for fractional IVPs. Commun. Nonlin. Sci. Numerical Simulat., 14: 674-684. DOI: 10.1016/j.cnsns.2007.09.014

Hesameddini, E. and F. Fotros, 2012. Solution for timefractional coupled Klein-Gordon Schrodinger equation using decomposition method. Int. Math. Foru., 7: 1047-1056.

Jumarie, G., 2009. Table of some basic fractional calculus formulae derived from a modified Riemann-Liouville derivative for non-differentiable functions. Applied Math. Lett., 22: 378-385. DOI: $10.1016 /$ j.aml.2008.06.003

Keskin, Y. and G. Oturanc, 2009. Reduced differential transform method for partial differential equations. Int. J. Nonlin. Sci. Num. Simul., 10: 741-749. DOI: 10.1515/IJNSNS.2009.10.6.741

Kilbas, A.A., H.M. Srivastava and J.J. Trujillo, 2010. Theory and Applications of Fractional Differential Equations. 1st Edn., Elsevier, Amsterdam, ISBN-10: 0444518320, pp: 523.

Klimek, K., 2005. Fractional sequential mechanicsmodels with symmetric fractional derivative. Czech. J. Phys., 55: 1447-1453. DOI: $10.1023 / \mathrm{A}: 1013378221617$

Khalil, H., R.A. Khan, M.H. Al-Smadi and A.A. Freihat, 2015a. Approximation of solution of time fractional order three-dimensional heat conduction problems with Jacobi Polynomials. J. Math., 47: 35-56.

Khalil, H., R.A. Khan, M.H. Al-Smadi, A.A. Freihat and N. Shawagfeh, 2015b. New operational matrix for shifted Legendre polynomials and fractional differential equations with variable coefficients. Pun. Uni. J. Math., 47: 81-103. 
Khalil, H., R.A. Khan, M. Al-Smadi and A. Freihat, 2015c. A generalized algorithm based on Legendre polynomials for numerical solutions of coupled system of fractional order differential equations. J. Fract. Calculus Applic., 6: 123-143.

Khader, M.M., N.H. Swetlam and A.M.S. Mahdy, 2014. The chebyshev collection method for solving fractional order Klein-Gordon equation. Wseas Trans. Math., 13: 31-38.

Komashynska, I. and M. Al-Smadi, 2014. Iterative reproducing kernel method for solving second-order integrodifferential equations of fredholm type. J. Applied Math., 2014: 459509-459519. DOI: $10.1155 / 2014 / 45950$

Komashynska, I., M. Al-Smadi, O. Abu Arqub and S. Momani, 2016. An efficient analytical method for solving singular initial value problems of nonlinear systems. Applied Math. Inform. Sci., 10: 647-656. DOI: 10.18576/amis/100224

Li, Z.B. and J.H. He, 2010. Fractional complex transform for fractional differential equations. Math. Comput. Applied, 15: 970-973.

Mainardi, F., 2010. Fractional Calculus and Waves in Linear Viscoelasticity. 1st Edn., World Scientific, ISBN-10: 1908978570, pp: 368.

Marasi, H.R. and S. Karimi, 2014. Convergence of the variational iteration method for solving fractional Klein-Gordon equation. J. Math. Comput. Sci., 4: 257-266.

Millar, K.S. and B. Ross, 1993. An Introduction to the Fractional Calculus and Fractional Differential Equations. 1st Edn., John Wiley and Sons, Inc., ISBN-10: 0471588849, pp: 384.

Moaddy, K., S. Momani and I. Hashim, 2011. The nonstandard finite difference scheme for linear fractional PDEs in fluid mechanics. Comput. Math. Applic., 61: 1209-1216. DOI: 10.1016/j.camwa.2010.12.072

Moaddy, K., M. AL-Smadi and I. Hashim, 2015. A novel representation of the exact solution for differential algebraic equations system using residual power-series method. Discrete Dynam. Nature Society, 2015: 205207-205218.

DOI: $10.1155 / 2015 / 205207$

Momani, S., A. Freihat and M. AL-Smadi, 2014. Analytical study of fractional-order multiple chaotic fitzhugh-nagumo neurons model using multistep generalized differential transform method. Abst. Applied Anal., 2014: 276279-276288.

DOI: $10.1155 / 2014 / 276279$
Odibat, Z. and S. Momani, 2006. Application of variational iteration method to nonlinear differential equations of fractional order. Int. J. Nonlin. Sci. Numer. Simul., 7: 27-34. DOI: $10.1515 /$ IJNSNS.2006.7.1.27

Ortigueira, M.D., 2010. The fractional quantum derivative and its integral representations. Commun. Nonlin. Sci. Numer. Simulat., 15: 956-962. DOI: 10.1016/j.cnsns.2009.05.026

Podlubny, I., 1999. Fractional Differential Equations. 1st Edn., Academic Press, San Diego, CA, USA.

Samko, S.G., A.A. Kilbas and O.I. Marichev, 1993. Fractional Integrals and Derivatives: Theory and Applications. 1st Edn., Gordon and Breach, New York.

Secer, A., 2012. Solving time-fractional reactiondiffusion equation by reduced differential transform method. Int. J. Comp. Sci. Emer. Tech., 3: 19-22.

Sohail, M. and S.T. Mohyud-Din, 2012. Reduced differential transform method for solving a system of fractional PDEs. Int. J. Mod. Math. Sci., 4: 21-29.

Srivastava, V.K., M.K. Awasthi and M. Tamsir, 2013. RDTM solution of Caputo time fractional-order hyperbolic Telegraph equation. AIP Adv., 3: 032142-032142. DOI: 10.1063/1.4799548

Srivastava, V.K., M.K. Awasthi and S. Kumar 2014. Analytical approximations of two and three dimensional time-fractional telegraphic equation by reduced differential transform method. Egypt. J. Basic Applied Sci., 1: 60-66. DOI: $10.1016 /$ j.ejbas.2014.01.002

Sweilam, N.H., M.M. Khader and A.M.S. Mahdy, 2012. On the numerical solution for the linear fractional Klein-Gordon equation using Legendre pseudospectral method. Int. J. Math. Comput. Applic. Res., 2: $1-10$.

Yang, A.M., Y.Z. Zhang, C. Cattani, G.N. Xie and M.M. Rashidi et al., 2014. Application of local fractional series expansion method to solve klein-gordon equations on cantor sets. Abst. Applied Anal., 2014: 372741-372746. DOI: 10.1155/2014/372741

Yusufoglu, E., 2008. The variational iteration method for studying the Klein-Gordon equation. Applied Math. Lett., 21: 669-674. DOI: 10.1016/j.aml.2007.07.023 